\title{
Trastornos de la alimentación: Anorexia nerviosa y bulimia nerviosa
}

\author{
Eating disorders: Anorexia nervosa and bulimia nervosa
}

\author{
Julián Andrés Hamdan Pérez ${ }^{1}$ \\ Andry Dilena Mela Botina ${ }^{2}$ \\ Margarita Pérez Hormiga ${ }^{3}$ \\ Beatriz Eugenia Bastidas ${ }^{4}$
}

\author{
Recibido: 02 de febrero 2017 \\ Revisado: 19 de abril 2017 \\ Aceptado: 29 de mayo 2017
}

\section{Resumen}

Los trastornos de la alimentación son enfermedades mentales crónicas que afectan principalmente a mujeres adolescentes; la depresión, la ansiedad y los medios de comunicación conllevan a las pacientes a modificaciones del hábito alimenticio para conseguir una "figura idónea" y ser socialmente aceptadas. La clasificación según criterios del DSM-V para estos trastornos de la alimentación son anorexia nerviosa (tipo restrictiva y tipo compulsiva/purgativa) y bulimia nerviosa (subclasificada según determinantes de gravedad basados en los episodios bulímicos por semana). La incidencia de estas enfermedades es baja, tienen poco registro epidemiológico y son subdiagnosticadas. Los principales determinantes psicológicos y físicos son la extrema preocupación por la comida, el miedo a aumentar de peso, una imagen corporal distorsionada, la deshidratación y una pérdida anormal de peso, que finalmente llevan al padecimiento de diversas complicaciones orgánicas como alteraciones hidroelectrolíticas y la desnutrición. Hoy en día existen diversos instrumentos que facilitan una detección temprana de conductas de riesgo, y otros que determinan el funcionamiento familiar, la depresión y ansiedad en estas personas. Su tratamiento es interdisciplinario, basado en la psicoterapia, en un manejo médico para las complicaciones orgánicas y un tratamiento psicofarmacológico encaminado a la comorbilidad psiquiátrica teniendo en cuenta que la bulimia nerviosa ha respondido mejor a los fármacos antidepresivos, mientras que en la anorexia nerviosa no existe un tratamiento farmacológico definitivo.

Palabras clave: Trastorno de la alimentación, anorexia nerviosa, bulimia nerviosa, adolescentes.

\begin{abstract}
Eating disorders are chronic mental illnesses that primarily affect teenage girls; depression, anxiety and the mass media lead to modifications of eating habits to achieve "ideal figure" and to be socially accepted. The classifications according to DSM-V for these eating disorders are anorexia nervosa (restrictive type and compulsive / purging type) and bulimia nervosa (subclassified as determinants of gravity based on the bulimic episodes per week). The incidence of these diseases is low, they have little epidemiological registry and are underdiagnosed. The main psychological and physical determinants are extreme preoccupation with food, fear of getting weight, a distorted body image, dehydration and abnormal weight loss; eventually leading to the occurrence of various organic complications such as electrolyte disturbances and malnutrition. Today, there are many ways to facilitate an early detection of risk behaviors and other determining family functioning, depression and anxiety in these people. This treatment is interdisciplinary, based on psychotherapy, in a medical management to organic complications and psychopharmacological treatment aimed at psychiatric comorbidity considering that bulimia nervosa has responded better to antidepressant drugs, while in anorexia nervosa there is not a definitive pharmacological treatment.
\end{abstract}

Key words: Eating disorder, anorexia nervosa, bulimia nervosa, teens.

\footnotetext{
${ }^{12}$ Estudiantes de Medicina. Facultad Ciencias de la Salud Universidad del Cauca.

${ }^{3}$ Psicóloga Clínica. Especialista en Educación e Intervención para la Primera Infancia. Universidad Cooperativa de Colombia. Fundación Amigos por una Nueva Colombia, Fundación Mujer Sola.

${ }^{4}$ MD. Especialista en Salud Familiar. Facultad Ciencias de la Salud Universidad del Cauca, Profesor titular Departamento de Medicina Social y Salud Familiar.

* Correspondencia: Julián Andrés Hamdan. Correo electrónico. julianhp101@hotmail.com
} 


\section{Introducción}

Los trastornos de la alimentación (TA), conocidos también como desórdenes alimenticios, son enfermedades mentales crónicas causadas principalmente por ansiedad, depresión, alteración de la percepción del peso y forma del cuerpo que conllevan a modificaciones en los hábitos alimenticios, presentadas principalmente en la adolescencia y asociadas a una alta tasa de mortalidad ${ }^{(1)}$

Los principales TA diagnosticados son anorexia nerviosa (AN) y bulimia nerviosa $(\mathrm{BN})$, de estas se desligan un amplio grupo de patologías influenciadas por factores biológicos, emocionales, psicológicos, interpersonales y sociales (2), compartiendo síntomas cardinales como insatisfacción de la imagen corporal y constante preocupación por la alimentación; lo anterior genera la ejecución de acciones poco saludables para controlar o reducir el peso, lo que a su vez compromete su bienestar psicosocial y físico ${ }^{(2)}$.

Los TA son patologías debilitantes, distinguidas por una alteración persistente en los hábitos alimenticios y conductas para controlar el peso, que causan complicaciones importantes en la salud ${ }^{(4)}$ y predisponen a otros comportamientos psiquiátricos como la autolesión y el abuso de sustancias ${ }^{(5)}$. Se consideran varios factores de riesgo que predisponen al desarrollo de algún trastorno de la alimentación, estos pueden ser biológicos, genéticos, psicológicos, socioculturales y/o medios de comunicación; éste último cobra importancia, ya que difunden un estereotipo falso de la belleza, destacando la sobrevaloración de la delgadez como signo de éxito y perfección ${ }^{(6)}$; frecuentemente estas personas niegan tener un problema y solo buscan ayuda de un profesional ante la insistencia de familiares y amigos.

Dada la importancia de la AN y la BN en el ámbito de la salud, se decidió hacer una revisión de tema sobre los aspectos relevantes de ésta problemática como su definición, clasificación, epidemiología, diferencia entre los tipos de TA, etiopatogenia, clínica, diagnóstico y tratamiento; con el fin de obtener información actualizada al respecto.

\section{Metodología}

Para la siguiente revisión, se recolectó bibliografía en libros de texto de medicina, psicología, tesis doctorales, artículos originales, artículos de revisión, información virtual en páginas web, y a través de los buscadores: PubMed, Proquest y Sciencie Direct utilizando los siguientes términos: "eating disorder", "anorexia nervosa", "bulimia nervosa". La búsqueda se limitó a información en inglés y español publicada entre enero del 2000 hasta noviembre del 2017. Se obtuvieron 92 publicaciones, de las cuales se seleccionaron 58 que aportaron información valiosa a ésta revisión.

\section{Definición de los trastornos de la alimentación}

Las personas con algún TA, dan un significado psicoafectivo específico al alimento de acuerdo a diversos factores psicológicos, biológicos y sociales, que se interrelacionan negativamente, convirtiendo la comida en el eje a partir del cual gira su vida y el mundo que los rodea.

Anorexia Nerviosa (AN): Es definida como privación de la ingesta de manera voluntaria que origina una pérdida excesiva de peso ${ }^{(7)}$. Las tres principales características de la AN, según Bruch ${ }^{(8)}$ son:

- Distorsión en la percepción de la imagen corporal ${ }^{(8)}$, es decir, la persona no tiene la capacidad de reconocer su delgadez.

- Percepción distorsionada de los estímulos propioceptivos: si la percepción de la realidad está distorsionada, también las emociones resultarán distorsionadas, serán exageradas o inadecuadas ${ }^{(8)}$.

- Sentimiento general de ineficacia personal: Geddie ${ }^{(9)}$ definió el término eficacia como el "poder de producir un efecto". La autoeficacia percibida por las personas que padecen algún TA es baja, en éstos pacientes prevalece el juicio de poder ejecutar pobremente las acciones requeridas y son incapaces de manejar situaciones potenciales; concepto que también ha sido utilizado indistintamente como impotencia y sentimiento de ineficacia.

Bulimia Nerviosa (BN): Se define como la ingesta excesiva de alimentos durante un periodo muy corto de tiempo, la persona recurre posteriormente a purgar su cuerpo vomitando, utilizando laxantes, enemas, diuréticos y/o ejercitándose excesivamente; a esto en un paciente ya diagnosticado se le denomina episodio bulímico compensatorio ${ }^{(7)}$. Los pacientes presentan insatisfacción corporal y algunos tienden a distorsionar su imagen corporal como sucede en la $\mathrm{AN}^{(10)}$.

\section{Clasificación de los trastornos de la ali- mentación según el DSM-V:}

El DSM-V es la quinta edición del Manual Diagnóstico y Estadístico de los Trastornos Mentales de la American Psychiatric Association, es una guía útil para la práctica clínica, debido a la claridad de expresión y la manifestación explícita de las hipótesis contenidas en los criterios diagnósticos ${ }^{(11)}$; ha sub-clasificado a la AN y determina la gravedad de la BN así:

\section{Anorexia Nerviosa (AN):}

La subclasificación de la anorexia nerviosa:

- Anorexia nerviosa tipo restrictiva: Se caracteriza por limitar severamente la ingesta de alimentos, principalmente aquellos de alto contenido calórico (carbohidratos y lípidos) para mantener un peso por debajo del índice de masa corporal que correspondería 
según su talla y edad(12). Identifican a este grupo el perfeccionismo, una mayor responsabilidad y la rigidez en sus hábitos alimenticios, además está acompañado de sentimientos de ineficacia que alimenta cada vez más su comportamiento para poder alcanzar el objetivo de ser una persona "socialmente aceptada"(13).

\section{- Anorexia nerviosa tipo compulsivo/purgativo: El individuo tiene restricción alimentaria por largos períodos de tiempo, regularmente presentan atracones, definidos como la pérdida del control al comer, es decir, consumir grandes cantidades de alimento en un corto periodo de tiempo ${ }^{(14)}$; posteriormente actos compen- satorios como la purga, aumentando su capacidad de restricción y creando un ciclo ${ }^{(15)}$. La inestabilidad diagnóstica es común en este tipo de $\mathrm{AN}$, puesto que pacientes con un diagnóstico inicial de AN tipo restric- tivo desarrollan síntomas compulsivo/purgativo du- rante su enfermedad, llegando a cruzar su diagnóstico a $\mathrm{BN}^{(16,17)}$.}

Adicionalmente, hay una tercera clasificación que cataloga la anorexia como una condición netamente clínica, atribuida a diversos factores etiológicos, como la apendicitis o en algunas enfermedades terminales que se acompañan de caquexia ${ }^{(14)}$.

\section{Bulimia Nerviosa (BN):}

La subclasificación de la bulimia nerviosa, según los determinantes de gravedad:

El DSM-V estableció grupos de gravedad según la frecuencia de los episodios compensatorios así: BN leve (1-3 episodios / semana), BN moderada (4-7 episodios / semana), BN grave (8-13 episodios / semana) y BN extrema (14 o más episodios / semana) ${ }^{(18)}$.

\section{Epidemiología}

Los trastornos de la alimentación ( $\mathrm{AN}$ y $\mathrm{BN}$ ) son consideradas como eventos infrecuentes y subdiagnosticados, por lo que es difícil establecer la incidencia y la prevalencia en la población general; y, aunque los criterios diagnósticos están bien definidos en el DSM-V y la Clasificación Internacional de Enfermedades (CIE-10), no son plenamente aplicables a niños y adolescentes, aumentando el sesgo en una recolección de datos ${ }^{(19)}$.

En el año 2012, un reporte de la Revista Nutrición Hospitalaria determinó que "la incidencia global de la AN, según criterios del DSM-IV, ha tenido tasas más estables en el tiempo. Las tasas de incidencia global se han mantenido estabilizadas cuando se evaluaron los años 80 y 90 (7,4/100.000/año para 19851989 y 7,7/ 100.000/año para 1995-1999); sin embargo cuando se evaluó la incidencia, teniendo en cuenta la edad, en el período 1995 a 1999, se vio un aumento significativo del $94 \%$ de AN en el grupo de niñas con edades comprendidas entre 15 y 19 años (56,4/100.000 personas/año para 109/ 100.000 personas/año"(19). También mostró resultados de un estudio en 2012 realizado en Reino Unido en donde "se identificó incremento global en la incidencia de $\mathrm{BN}$ en el período 1998 a 2000. Cabe destacar que este aumento fue más significativo en los primeros años de la década del 90, con un pico en 1996 con la subsiguiente disminución de alrededor del 38,9\% hasta el año 2000. Unos investigadores registraron tasas de incidencia global similares en los años 90, con 6,1/100.000/año y el año 2000, con 6,6/100.000/año"(19).

A nivel mundial, en el año 2013 la prevalencia en doce meses de AN entre mujeres jóvenes es de aproximadamente $0,4 \%$; en el sexo masculino la prevalencia es difícil de calcular debido a una proporción muy pequeña aunque actualmente se ha visto un aumento en la incidencia de estos trastornos. En poblaciones clínicas se refleja una proporción de aproximadamente 1:10 relación hombre mujer ${ }^{(11)}$, esto no quiere decir que estas patologías sean privativas del género femenino, la dificultad radica en la identificación y aceptación de los hombres frente a un trastorno alimenticio debido a la estigmatización de ser únicamente femeninas; por otro lado, si un hombre acepta un problema de imagen corporal pocas veces será diagnosticado con estos trastornos por parte del profesional ${ }^{(20)}$.

En cuanto a la BN, la prevalencia en mujeres durante el año 2013 es de aproximadamente 1 - 1,5\%, siendo más alta entre adultos jóvenes, ya que los picos del trastorno se presentan principalmente en la adolescencia y en la edad adulta; éste dato es más difícil de identificar en hombres, pero se conoce que es menos común que en las mujeres ${ }^{(11)}$.

Teniendo en cuenta lo anterior, las mujeres parecen ser vulnerables al desarrollo de trastornos alimenticios: estudios indican que éste sexo representa el 90\% de los diagnósticos, siendo la edad entre 15 a 19 años el subgrupo poblacional con mayor riesgo ${ }^{(21)}$, en el cual los factores socioculturales desempeñan un papel significativo en la patogenia de estos trastornos ${ }^{(22)}$.

Otros estudios muestran que atletas universitarias tienen menor prevalencia de TA y menos dificultades con la regulación de sus emociones depresivas que las no atletas; las atletas están protegidas de éstos desórdenes debido a un comportamiento alimenticio distinto, que se debe a otros atributos relacionados con su estado físico. La participación en deportes es un predictor significativo de baja incidencia en estos desórdenes independientemente de los antecedentes familiares con TA; sin embargo, al no practicar o ejercer alguna actividad física, la historia familiar de estos trastornos quintuplica el riesgo de padecimiento ${ }^{(23)}$.

Por último, existe poco registro epidemiológico de éstas enfermedades a nivel nacional y local, ya que su presentación en la población general es realmente baja, no todas las personas que las padecen acuden a especialistas y convierten estos TA en enfermedades subdiagnosticadas. 


\section{Diferencias entre anorexia nerviosa y bulimia nerviosa}

La AN y BN han causado cierta confusión, puesto que los comportamientos de estos trastornos son muy similares; sin embargo, la diferencia radica en la conducta ante la comida.

En la AN encontramos un miedo exagerado a aumentar de peso y se caracteriza por distorsión de la imagen corporal, estas personas manejan un peso por debajo de lo normal, tienen mayor control, orden, introversión, obsesiones y ansiedad.

En contraste, la BN no maneja distorsión corporal, presentando un peso aproximado al normal. El descontrol, impulsividad, depresiones y fluctuaciones rápidas en el estado de ánimo hacen parte del cuadro patológico de este trastorno ${ }^{(24,25)}$.

\section{Etiopatogenia}

La etiología de los trastornos de la alimentación sigue siendo confusa; sin embargo, dentro del campo psiquiátrico se han señalado como trastornos multifactoriales que se fundamentan en 3 causas principales: factores biológicos, psicológicos y socioculturales ${ }^{(26)}$.

- Factores biológicos: El eje hipotalámico-pituitariosuprarrenal (HPS) desempeña un papel importante en los TA, este es un regulador del apetito, de la tensión y del humor a través de los neurotransmisores tales como la serotonina, importante en la ansiedad y el apetito; la noradrenalina, un regulador de la tensión y la dopamina, significativa en la estimulación del sistema de recompensa. Un desequilibrio entre serotonina y dopamina explica por qué la gente con AN no deriva sensación de placer en la comida y de otras comodidades comunes ${ }^{(27)}$.

En algunas familias en las que se haya diagnosticado algún TA, es frecuente encontrar otros miembros con una patología similar, de forma evidente o silenciada; por eso es importante que se realice una buena anamnesis de la familia para determinar conductas de riesgo y/o la patología como tal.

Algunos estudios confirman que los familiares de pacientes con TA tienen de cuatro a cinco veces más probabilidades que la población general de desarrollar estos trastornos, principalmente $\mathrm{BN}^{(26)}$. Cabe resaltar que la familia cumple un papel importante durante el tratamiento, debido a su complicidad en los comportamientos de estas patologías ${ }^{(28)}$.

Factores psicológicos: Los aspectos relevantes desde el punto de vista psicológico son: la imagen corporal, autoestima, alexitimia, la sintomatología depresiva y ansiosa en estos desórdenes alimenticios ${ }^{(29)}$.
La imagen corporal: La ideología del cuidado nutricional para llevar un idóneo de imagen corporal, hace circular diversas informaciones acerca del comer, el ejercicio y las siluetas saludables; sin embargo, la información puede tomar caminos equivocados que llevan a la enfermedad, con el objetivo de alcanzar dicho idóneo ${ }^{(30)}$. La imagen corporal incluye dos componentes: el primero es el perceptivo, que hace referencia a la estimación de la apariencia, y el segundo es el actitudinal, que recoge los sentimientos y actitudes hacia el propio cuerpo; evidentemente estos componentes se encuentran alterados en los TA.

El trastorno de la imagen corporal incluye aspectos de tipo perceptivos, afectivos y cognitivos, en el cual la preocupación exagerada por el físico, lleva a devaluar la apariencia y preocuparse en exceso por la opinión de los demás; por ello, las personas con éstos trastornos ocultan su cuerpo, lo someten a dietas, ejercicios y comportamientos inadecuados para conseguir ese ideal, quieren ser "aceptados socialmente" pero se alejan paradójicamente del componente social mientras cumplen su objetivo ${ }^{(31)}$.

Autoestima: Definida como auto-percepción, siendo una de las funciones psicológicas más importantes en la vida de las personas; se desarrolla a partir de la interacción humana, en la que el YO evoluciona por medio de pequeños logros, reconocimientos y el éxito ${ }^{(32)}$.

La autoestima es de gran importancia en estos cuadros por su asociación directa con estrés, depresión, trastornos de personalidad, ansiedad, fobia social y trastornos de la conducta alimentaria ${ }^{(33)}$. La baja autoestima es uno de los detonantes para que personas con inconformismo en su imagen corporal busquen continuamente la aprobación de los demás; en los TA se da un deterioro de los factores que conforman una autoestima sana: autoconcepto, autorespeto y autoaceptación $^{(34)}$.

Alexitimia: Trastorno cognitivo en el cual la persona tiene gran dificultad para verbalizar estados afectivos e identificar emociones propias (hay ausencia de la autoevaluación), puede aparecer en personas con una amplia variedad de diagnósticos clínicos como en el autismo o en los TA. Aunque este término es aceptado por la comunidad científica no aparece como constructo en las clasificaciones psiquiátricas dada por el DSM-V ${ }^{(35)}$.

Síntomas depresivos y de ansiedad: Los síntomas depresivos se pueden manifestar como el hecho de sentirse triste, melancólico, infeliz, abatido o derrumbado ${ }^{(36)}$. La depresión ha sido vista como uno de los padecimientos que más se relaciona con los TA, principalmente con $\mathrm{BN}^{(37)}$.

La ansiedad es el resultado de un proceso neurótico manifestado a través de cambios del estado de ánimo, que ponen al individuo en un estado de alerta frente a situaciones de peligro que no tienen fundamento real 
aparente, asociado a esto se presentan manifestaciones tanto somáticas como subjetivas: taquicardia, diarrea, náuseas, disnea; además de trastornos del sueño, miedo, inseguridad y sensación de desesperación, suele evidenciarse como disforia (mezcla de ansiedad, irritabilidad y tristeza) en los TA. ${ }^{(38)}$

\section{- Factores socioculturales}

Hoy en día, el impacto de los medios de comunicación respecto al "idóneo" de imagen corporal, puede ser un factor detonante para inculcar la idea de convertirse en alguien "socialmente aceptado", cultivando la idea de la delgadez al ser comparada con el éxito y el renombre, siendo afectadas principalmente las mujeres ${ }^{(27)}$.

La imagen corporal no es estática, más bien es un constructo dinámico, que varía a lo largo de la vida en función de las propias experiencias, pero sobre todo, de la estigmatización social que se tenga en la época o momento, con influencias sociales y culturales que matizan la autopercepción del cuerpo desde el nacimiento ${ }^{(39)}$.

Los factores socioculturales en la región occidental proponen un ideal estético que se relaciona con un "alto autoestima" comparándolo con el atractivo físico y la competencia personal (lo que es bello es bueno: estigmatización en una silueta perfecta con repulsión a la gordura como un estilo de vida ideal) y como ya se mencionó, mientras que en occidente se habla de una "epidemia de casos incidentes en TA", en los países del tercer mundo y en las áreas no occidentalizadas en vías de desarrollo apenas se conocen estas patologías ${ }^{(40)}$.

\section{Signos y síntomas}

En el desarrollo de estos trastornos (anorexia nerviosa y bulimia nerviosa) se presenta una serie de comportamientos peculiares de excesiva preocupación por la imagen corporal y cambios en la alimentación habitual, como el rechazo por algunos alimentos y dietas extremas $^{(41)}$. Es necesario que se detecten algunos determinantes psicológicos claves, como la preocupación por la comida, el miedo a aumentar de peso, la negativa a comer, el ejercicio compulsivo, una imagen corporal distorsionada, el comer solo o en secreto, el abuso de laxantes, diuréticos, pastillas para adelgazar y/o eméticos, los síntomas depresivos, la vergüenza y la culpa; y determinantes físicos como la deshidratación, el pelo fino y excesivo en cuerpo, la pérdida anormal de peso, la sensibilidad al frío y la amenorrea.
Estos trastornos de la alimentación conllevan a un deterioro progresivo a nivel orgánico; dentro de los hallazgos clínicos el evaluador puede encontrar alteraciones en los siguientes sistemas:

Sistema nervioso central: apatía, aburrimiento, hipoprosexia o disprosexia, desregulación emocional y asociado a estos cuadros se pueden diagnosticar ciertas comorbilidades de los TA como depresión, trastornos de ansiedad, trastorno obsesivo-compulsivo e incluso puede llevar al abuso de sustancias.

Cardiovascular: pulso débil e irregular, hipotensión, mareos, lipotimia, sincopes, falta de aliento, angina, hipokalemia y desequilibrios electrolíticos (por vómitos y baja ingesta) que pueden inducir arritmias o un paro cardíaco potencialmente mortal.

Musculo-esqueléticos: retraso del crecimiento en niñas(os), fracturas y/o osteoporosis.

Gastrointestinal: erosiones en boca, pérdidas dentales, hipertrofia de glándulas salivales, pirosis, hematemesis por desgarros en esófago (Síndrome de Mallory-Weiss) y/o ruptura del esófago que puede conducir a un colapso circulatorio y la muerte, ruptura gástrica por émesis inducida (tasa de mortalidad del 80\%), peristaltismo reducido debido a dietas restrictivas, estreñimiento frecuente con evacuaciones irregularmente crónicas.

Sistema endocrino: alteración de la tiroides, intolerancia al frio, temperatura corporal baja, pérdida de peso, pérdida de cabello y/o aumento de vello corporal.

\section{Complicaciones asociadas al abuso de laxantes:} insuficiencia renal, colon catártico (incapacidad intestinal de funcionar normalmente sin el uso de grandes dosis de laxantes, debido a la destrucción de los nervios en el colon que controlan la eliminación), desequilibrio electrolítico, deshidratación y/o dependencia ${ }^{(42)}$.

\section{Criterios diagnósticos según el DSM-V}

Los criterios diagnósticos para la anorexia nerviosa y la bulimia nerviosa determinan una serie de signos y síntomas que permiten una aproximación en el diagnóstico de cierta patología para la que no se describen datos patognomónicos ${ }^{(43)}$ (tabla 1 ). 
Tabla 1. Criterios diagnósticos según el DSM-V para anorexia nerviosa y bulimia nerviosa

\begin{tabular}{|c|c|}
\hline \multirow{3}{*}{ Anorexia Nerviosa } & $\begin{array}{l}\text { Criterio A: Restricción del consumo energético } \\
\text { relativo a los requerimientos que conlleva a un peso } \\
\text { corporal marcadamente bajo. Un peso marcadamente } \\
\text { bajo es definido como un peso que es inferior al mínimo } \\
\text { normal o, para niños y adolescentes, inferior a lo que } \\
\text { mínimamente se espera para su edad y estatura. }\end{array}$ \\
\hline & $\begin{array}{l}\text { Criterio B: Miedo intenso a ganar peso o a convertirse } \\
\text { en obeso, o una conducta persistente para evitar ganar } \\
\text { peso, incluso estando por debajo del peso normal. }\end{array}$ \\
\hline & $\begin{array}{l}\text { Criterio C: Alteración de la percepción del peso o la } \\
\text { silueta corporal, exageración de su importancia en la } \\
\text { autoevaluación o persistente negación del peligro que } \\
\text { comporta el bajo peso corporal actual. }\end{array}$ \\
\hline \multirow{5}{*}{ Bulimia Nerviosa } & $\begin{array}{l}\text { Criterio A: Presencia de atracones recurrentes. Un } \\
\text { atracón se caracteriza por: } \\
\text { - Ingesta de alimentos en un corto espacio de tiempo } \\
\text { (ej. dos horas) en cantidad superior a la que la } \\
\text { mayoría de las personas ingerirían en un período de } \\
\text { tiempo similar y en las mismas circunstancias. } \\
\text { - Sensación de pérdida de control sobre la ingesta de } \\
\text { alimentos (ej. no poder parar de comer o no poder } \\
\text { controlar el tipo o cantidad de comida que se está } \\
\text { ingiriendo). }\end{array}$ \\
\hline & $\begin{array}{l}\text { Criterio B: Conductas compensatorias inapropiadas, } \\
\text { de manera repetida, con el fin de no ganar peso, como } \\
\text { son provocación del vómito, uso excesivo de laxantes, } \\
\text { diuréticos, enemas u otros fármacos, ayuno y ejercicio } \\
\text { excesivo. }\end{array}$ \\
\hline & $\begin{array}{l}\text { Criterio C: Los atracones y las conductas compensa- } \\
\text { torias inapropiadas tienen lugar como promedio al menos } \\
\text { dos veces a la semana durante un período de } 3 \text { meses. }\end{array}$ \\
\hline & $\begin{array}{l}\text { Criterio D: Autoevaluación exageradamente influida } \\
\text { por el peso y silueta corporales. }\end{array}$ \\
\hline & $\begin{array}{l}\text { Criterio E: La alteración no aparece exclusivamente } \\
\text { durante episodios de anorexia nerviosa. }\end{array}$ \\
\hline
\end{tabular}

Cuestionarios, inventarios y escalas para los trastornos de la alimentación

Desde los años 90, el campo de la psicología ha mostrado gran interés por desarrollar diversos instrumentos como cuestionarios, inventarios y escalas que permitan medir el impacto biopsicosocial de la imagen corporal en éstos TA.

En la actualidad, hay diversos instrumentos que permiten evaluar las rutinas alimentarias, la percepción de la imagen corporal, ansiedad, depresión, autoestima y cuestionarios nutricionales ${ }^{(4)}$. Todos estos instrumentos que facilitan una exploración o confirman un diagnóstico frente a los TA, han sido traducidos y val- idados para países con culturas y personalidades muy distintas a las de habla inglesa, teniendo en cuenta que el lenguaje es un factor determinante en la aplicación e interpretación de estos, debido a que la semántica varía al traspasar las fronteras culturales ${ }^{(45)}$.

Actualmente existen diversos instrumentos que valoran éste tipo de patologías, pero se mencionarán los más importantes:

\section{Instrumentos de valoración psicométrica}

Estos instrumentos valoran la comorbilidad, permitiendo realizar una medición de las capacidades psíquicas del individuo, explorando principalmente los 
hábitos alimenticios y la imagen corporal $^{(44)}$.

El Eating Attitudes Test (EAT-40), elaborado por Garner y Garfinkel en el año 1979, valora las conductas alimentarias, cuyo objetivo principal es una medición

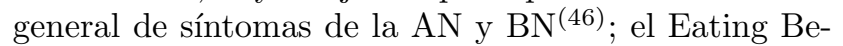
haviours and Body Image Test for Preadolescent Girls (EBBIT) realizado por Candy y Fee en 1998, indica posibles síntomas de los $\mathrm{TA}^{(44)}$; el cuestionario Sick Control on Fat Food (SCOFF) de Morgan, Reid y Lacey en 1999, es un tamizaje para detectar signos sugestivos de TA, ha sido diseñado para sugerir más no para diagnosticar patologías ${ }^{(47)}$; el Eating Disorder Inventory-2 (EDI-2) elaborado por Garner en 1991, es una escala de auto-reporte que mide aspectos psicológicos y síntomas asociados con éstas patologías ${ }^{(45)}$; el Food Craving Questionnaire-Trait y Food Craving Questionnaire-State (FCQ-T, FCQ-S) realizados por Cepeda-Benito en el año 2000, miden el food craving (ansia por la comida) como rasgo, deseo normal de la persona por la comida; y estado, deseo de la persona por la comida en un momento dado ${ }^{(44)}$.

\section{Instrumentos para valoración de la imagen cor- poral}

El concepto de imagen corporal es muy amplio, en el que intervienen elementos perceptivos, actitudinales y emocionales. Se han desarrollado dos tipos de instrumentos que evalúan alteraciones de la percepción de la imagen corporal: evaluación gráfica (distorsión de fotografías, videos o evaluación de siluetas) y evaluación mediante cuestionarios ${ }^{(48)}$.

El Body Shape Questionnaire (BSQ) de Cooper de Taylor y Fairburn, 1987, mide de manera objetiva la preocupación del individuo por su peso y la imagen corporal, funciona como una herramienta de tamizaje en individuos que están en riesgo de padecer algún trastorno de la conducta alimentaria ${ }^{(49)}$, y al igual que éste, existen otros cuestionarios como el Body Attitudes Test (BAT) de Coppenolle, Probst, Vandereycken, Goris y Meerman, 1984; el Body-Self Relations Questionnaire (BSRQ) de Winstead y Cash, 1984; el Body Image Anxiety Scale (BIAS) de Reed, Thompson, Brannick y Sacco, 1990, entre otros, que finalmente evalúan aspectos importantes sobre la imagen corporal $^{(44)}$.

\section{Instrumento de valoración psicológica para el clima familiar}

El clima familiar se define como la percepción común que tienen padres e hijos acerca del funcionamiento familiar, desde la intensidad de los conflictos hasta el grado de cohesión afectiva entre los mismos; es importante saber que la familia sufre los efectos del trastorno y el prolongado tratamiento del mismo, del cual no siempre se obtienen buenos resultados ${ }^{(50)}$. La Escala de Clima Familiar (FES) de Moos, 1986, es tal vez el cuestionario más usado hoy en día para valorar éste aspecto, valora las características socio-ambientales de todo tipo de familias mediante tres dimensiones: cohesión, expresividad y conflicto ${ }^{(51)}$.

\section{Instrumentos para valoración de la depresión y ansiedad}

Como ya se ha comentado, éstos son elementos importantes en el desarrollo de los TA, uno de los test más usados es la Escala de Autoevaluación para la Depresión de Zung (SDS) de Conde y Cols, 1970; se trata de una encuesta de auto-aplicación que consta de 20 preguntas, en la que se valora cuatro aspectos comunes en la depresión: el efecto dominante, los equivalentes fisiológicos, otras perturbaciones y las actividades psicomotoras ${ }^{(52)}$, y para la ansiedad es usado el State-Trait Anxiety Inventory (STAI) de Spielberger, 1970 que mide ansiedad-estado y ansiedad-rasgo, dentro de los resultados se determina finalmente si el paciente tiene baja, moderada o elevada ansiedad ${ }^{(44)}$.

\section{Tratamiento}

El tratamiento consiste en psicoterapia enfocada al trastorno y corrección de las afecciones biológicas secundarias, siendo un procedimiento interdisciplinar que comprende intervención médica especializada, rehabilitación nutricional, consejería nutricional, psicoterapia individual e intervenciones en familia ${ }^{(53,54)}$. Estos trastornos de la alimentación incluyen aspectos psicofisiológicos, por tal razón, los tratamientos deben combinar la experiencia de la psicoterapia (enfocados en depresión y ansiedad) con profesionales de salud nutricional, que integren los efectos fisiológicos de estos ${ }^{(55)}$.

Dentro de los aspectos dietético-nutricionales del tratamiento, es importante la recuperación del peso, en la que se incluye la corrección de la baja ingesta, la concientización a los pacientes de una dieta saludable y balanceada y un plan dietético personalizado basado en sus necesidades nutricionales, para esto, es relevante el diagnóstico precoz y la intervención nutricional desde el comienzo ${ }^{(56)}$.

La terapia cognitivo-conductual para los TA difiere entre los pacientes, el curso del tratamiento debe ser de 16 a 20 sesiones en un período aproximado de 4 a 5 meses en adultos, y en adolescentes se adapta la terapia acorde a su edad, circunstancias y nivel de desarrollo, en el cual debe incluirse la familia según corresponda $^{(57)}$.

Lo estudios han demostrado que los medicamentos son más útiles en $\mathrm{BN}$, mientras que han sido decepcionantes en la AN. Se han realizado muchos ensayos de medicamentos en pacientes con TA, con resultados significativos, en particular con medicamentos antidepresivos como los inhibidores selectivos de la recaptación de serotonina (ISRS) y antidepresivos tricíclicos (ATC). 
Los ISRS se consideran a menudo agentes de primera línea para el tratamiento de la BN, dentro de los cuales, la fluoxetina es el único fármaco aprobado por la FDA (Food and Drug Administration) por sus efectos secundarios favorables, destacando un efecto neutral en el peso, mientras que los ATC se utilizan con menos frecuencia, en parte, por los efectos adversos como sedación, estreñimiento y la ganancia de peso; sin embargo, el uso de ISRS ha aumentado el riesgo de suicido en los pacientes más jóvenes, por lo tanto es imperativo que los médicos controlen y vigilen los riesgos que tienen los pacientes y sus familias.

El ensayo clínico controlado más grande que se ha realizado para comprobar la eficacia de la fluoxetina en los TA fue realizado durante 8 semanas, en 13 sitios aleatorios, en donde se comparó la eficacia de $60 \mathrm{mg} /$ día y $20 \mathrm{mg} /$ día de fluoxetina con el placebo. Levine et al. encontraron que $60 \mathrm{mg} /$ día fue superior al placebo y 20 $\mathrm{mg} /$ día ayudaba a reducir la frecuencia de atracones y purgas, y basándose en estos resultados, los antidepresivos se prescriben en dosis más altas en el tratamiento de la BN comparado con el tratamiento de la depresión.

Además de la fluoxetina, muchos otros antidepresivos han sido útiles en el tratamiento de la $\mathrm{BN}$, incluyendo otros ISRS (sertralina, fluvoxamina, y citalopram), ATC (imipramina, inhibidores de la desipramina y amitriptilina) y de la monoamino oxidasa (fenelzina).

El topiramato es un anticonvulsivo asociado con efectos sobre el apetito que también ha sido estudiado por su utilidad en la $\mathrm{BN}$; en un ensayo de 10 semanas en 64 pacientes ambulatorios con este trastorno, se administró topiramato (mediana de dosis de 100 mg/día) en comparación con placebo en donde hubo una reducción significativa de los atracones y purga, así como la mejora en medidas psicológicas, sin embargo, se ha informado que algunos pacientes pueden tener una pérdida significativa de peso, lo que puede complicar el tratamiento y, en estos casos, estaría contraindicado su uso.

Muchos medicamentos han sido considerados para el tratamiento de la AN sin tener mayor efectividad, cuyo fracaso farmacológico puede explicarse por su estado de desnutrición, por esta razón, la farmacoterapia no es el tratamiento de elección, sin embargo, cabe resaltar que muchas personas con este trastorno reciben medicamentos como parte de su plan de tratamiento, especialmente aquellos que no responden a la psicoterapia o la rehabilitación nutricional.

Los síntomas de la AN se superponen con otros trastornos psiquiátricos, incluida la depresión mayor, ansiedad generalizada y trastorno obsesivo-compulsivo. Por esta razón, los antidepresivos fueron considerados como una opción terapéutica prometedora para la AN, sin embargo, sorprendentemente estos medicamentos como la fluoxetina no han sido mejor que el placebo en cuanto a cambios en el peso o cualquier síntoma psicológico asociado.

Debido a los altos grados de ansiedad y cogniciones rígidas del paciente con $\mathrm{AN}$, los medicamentos antipsicóticos han sido considerados para su tratamiento. El desarrollo de los antipsicóticos de segunda generación (ASG) han demostrado resultados prometedores, de éstos, la olanzapina es la más prometedora, la cual se ha usado recientemente para incrementar el Índice de masa corporal en pacientes ambulatorios con AN, y se recomienda su uso en combinación con otros métodos terapéuticos ${ }^{(58)}$.

\section{Conclusiones}

La incidencia de la $\mathrm{AN}$ y la $\mathrm{BN}$ a nivel mundial es realmente baja, y aunque las mujeres adolescentes son las que más padecen estos trastornos, pocas acuden al médico para ser diagnosticadas bajo los criterios del DSM-V y recibir tratamiento según corresponda, de modo que se determina un bajo registro epidemiológico para éstas patologías. Los diversos estudios que se han realizado señalan que dentro de los aspectos biológicos, psicológicos y socioculturales que influyen en éstas enfermedades, están la depresión, ansiedad y medios de comunicación como los principales factores etiológicos que se interrelacionan entre sí, para el desarrollo de éstos TA.

La extrema preocupación por la comida, el miedo a aumentar de peso, la negativa a comer, una imagen corporal distorsionada, la deshidratación y una pérdida anormal de peso son los principales determinantes psicológicos y físicos que se pueden encontrar en éstas patologías, aparte de los diversos signos, síntomas y complicaciones que conllevan éstas enfermedades como: síncopes, hipotermia, hematemesis, alteraciones dentales, arritmias cardiacas, colon catártico por abuso de laxantes y alteraciones hidroelectrolíticas, entre otras.

Hoy en día, existen diversos instrumentos que nos facilitan una detección temprana de conductas de riesgo que el paciente puede tener, y que van encaminadas en el desarrollo de éstos TA, así como cuestionarios para determinar el funcionamiento familiar, la depresión y ansiedad en éstas personas.

El tratamiento de éstas enfermedades es interdisciplinario: psicoterapia enfocada en depresión y ansiedad; adecuado proceso nutricional; controles médicos con énfasis en las alteraciones físicas y orgánicas y finalmente un tratamiento psicofarmacológico, encaminado tanto a la comorbilidad psiquiátrica como al estado de ánimo del paciente. Los antidepresivos como los ISRS son clínicamente útiles en el tratamiento de la $\mathrm{BN}$ a diferencia de la $\mathrm{AN}$, en donde existe un mayor desafío debido a que los medicamentos no ofrecen ningún beneficio significativo. La olanzapina parece tener un efecto mínimo pero significativo en el aumento de peso en la $\mathrm{AN}$, sin embargo no debe ser utilizado como un tratamiento 
independiente.

\section{Referencias}

1. Guido KW Frank. What causes eating disorders, and what do they cause?. Biopsych. 2015; 77(7):60203 .

2. Neda Feeding Hope [Internet]. EE.UU: La asociación; s.f. [citado 3 Ago 2015] Información generalTipos y síntomas de los Trastornos alimenticios [aprox. 2 pantallas]. Disponible en:

https://www.nationaleatingdisorders.org/generalinformation.

3. Lopez C, Treasure J. Trastornos de la Conducta Alimentaria en Adolescentes: Descripción Y Manejo. Rev Med Clin Condes. 2011; 22(1): 85-97.

4. Camarillo Ochoa N, Cabada Ramos E, Gómez Méndez A, Munguía Alamilla K, Prevalencia de trastornos de la alimentación en adolescentes, Rev Esp Méd Quir, 2013; 18: 51-5.

5. Micali N. Solmi F. Horton N. Crosby R. Eddy K. Calzo J. Sonneville K. Swanson S. Field A. Adolescent eating disorders predict psychiatric, high-risk behaviors and weight outcomes in young adulthood. AACAP. 2015; 54(8): 652-59.

6. Instituto Tomás Pascual Sanz. Vive sano. trastornos del comportamiento alimentario: anorexia y bulimia nerviosas. Madrid: instituto Tomás Pascual Sanz; 2011.

7. American Psychological Association. [Internet]. Washington,DC: The Association; c2015 [citado 7 Ago 2015] trastornos de la alimentación. Disponible en: http://www.apa.org/centrodeapoyo/trastornos.aspx

8. Psicocode. Psicología y Desarrollo Personal. Trastornos alimentarios. Torrevieja: Psicocode; s.f.

9. Behar R. Sentimientos de ineficacia personal en los trastornos de la conducta alimentaria. Rev. Mex. de trastor. Aliment. 2011; 2: 113-24.

10. Bosque-Garza J, Caballero-Romo A. Consideraciones psiquiátricas de los trastornos de la conducta alimentaria: anorexia y bulimia. Bol. Med. Hosp. Infant. Mex, 2009; 66(5): 398-409.

11. American Psychiatric Association. Feeding and Eating Disorders. En: Narrow W, Regier D, Schultz SK, et al. editores. DSM-V. Diagnostic and Statistical Manual of Mental Disorders. 5a Edición. WashigtonLondon-England: American Psychiatric Publishing; 2013. p. $338-50$.

12. American Psychological Association. [Internet]. España: The Association; c2015 [citado 22 Ago 2015]
Síntomas de la anorexia-Características básicas de la anorexia [aprox. 4 pantallas]. Disponible en:

http://www.innatia.com/s/c-anorexia-nerviosa/asintomas-de-la-anorexia.html.

13. Espina A, Ortego MA, Ochoa de Alda I, Yenes F, Alemán A. La imagen corporal en los trastornos alimentarios. Psicothema. 2001; 13 (4): 533-38.

14. Elran-Barak R, Sztainer M, Goldschmidt A, Crow S, Peterson C, Hill L, et al. Dietary Restriction Behaviors and Binge Eating in Anorexia Nervosa, Bulimia Nervosa and Binge Eating Disorder: Trans-diagnostic Examination of the Restraint Model. Eat Behav. 2015; 18: $192-96$.

15. Elran-Barak R, Accurso E, Goldschmidt A, Sztainer M, Byrne C, Le Grange D. Eating patterns in Youth with restricting and binge eating/purging type anorexia nervosa. Int J Eat Disord, 2014; 47(8): 878-83

16. Monteleone P, Di-Genio M, Monteleone A, DiFilippo C, Maj M. Investigation of factors associated to crossover from anorexia nervosa restricting type (ANR) and anorexia nervosa binge-purging type (ANBP) to bulimia nervosa and comparison of bulimia nervosa patients with or without previous ANR or ANBP.Compr Psychiatry. 2011; 52(1): 56-62.

17. Olatunji B, Se-Kang K, Wall D. Extracting body image symptom dimensions among eating disorder patients: the profile analysis via multidimensional scaling (pams) approach. Bod Imag. 2015; 15:16-23.

18. Grilo C, Ivezaj V, White M. Evaluation of the DSM-5 severity indicator for bulimia nervosa. Behav Res Ther. 2015; 67: 41-4.

19. Portela de Santana M, Costa-Ribeiro H, MoraGiral M, Raich R. La epidemiología y los factores de riesgo de los trastornos alimentarios en la adolescencia; una revisión. Rev. Nutr Hosp. 2012; 27(2): 391-401.

20. García Sandoval C. Anorexia y bulimia en varones adolescentes: Factores de riesgo. RPC [serial en internet: ISSN 2322-8644], 2009 [citado 27 ago 2015]; 11(12), [aprox. 4 p.] Disponible en:

http://www.psicologiacientifica.com/anorexiabulimia-varones-adolescentes-factores-de-riesgo/

21. Smink F. Hoeken D. Hoek H. Epidemiology of eating disorders: incidence, prevalence and mortality rates. Curr psychiatry rep. 2012; 14(4): 406-14.

22. Toomey C, DiMartini J. Trastornos alimenticios. David-Elkin G (ed.) Psiquiatria Clínica. $1^{\mathrm{a}}$ ed. México: Editorial Mcgraw Hill Interamericana; 2000. p. 211-21.

23. Wollenberg G, Shriver L, Gates G. Comparison of disordered eating symptoms and emotion regulation 
difficulties between female college athletes and nonathletes. Eat Behav. 2015; 18: 1-6.

24. No a la obesidad [Internet]. México D.F.: la asociación; s.f. [citado 20 ago 2015]. Diferencia entre anorexia y bulimia [aprox. 4 pantallas]. Disponibles en:

http://www.noalaobesidad.df.gob.mx/index.php?opti on $=$ comcontent $\&$ view $=$ article $\& i d=53 \&$ Itemid $=64$.

25. Centro de Psicología López de Fez [Internet]. Valencia: La asociación; s.f. [citado 20 ago 2015]. Diferencias entre anorexia y bulimia [aprox. 3 pantallas]. Disponible en:

http://www.centropsicologialopezdefez.es/diferenciasentre-anorexia-y-bulimia/2-89-31-89.htm.

26. Siquia [Internet]. España: La asociación; c2013 [citado 7 Ago 2015]. Causas de los trastornos alimentarios [aprox. 3 pantallas]. Disponible en: http://www.siquia.com/2013/10/causas-trastornosalimentarios/

27. News Medical [Internet].EE.UU: La asociación; s.f. [Actualizado 15 Oct 2013; citado 8 Ago 2015]. ¿Qué Causa Trastornos Alimentarios? [aprox. 3 pantallas]. Disponible en:

http://www.news-medical.net/health/What-Causes-

Eating-Disorders-(Spanish).aspx

28. Grange D. Lock J. Loeb K. Nicholls D. Academy for eating disorders position paper: The role of the family in eating disorders. Int $\mathrm{j}$ of eat disorder. 2010; $43(1): 1-5$

29. Sanchez J. Un modelo estructural de conducta alimentaria de riesgo en adolescentes escolarizados. Tesis doctoral. Monterrey: Universidad Autónoma de Nuevo León, Facultad de Psicología; 2009.

30. Red de Salud UC CHRISTUS [Internet]. Chile: La asociación; c2014 [citado 7 Ago 2015]. Unidad de Trastornos de la Alimentación [aprox. 3 pantallas]. Disponible en:

http://redsalud.uc.cl/ucchristus/psiquiatria/Unidades /unidaddetrastornosdelaalimentacion.act.

31. AECC contra el cáncer [Internet]. España: La asociación; c2015 [Actualizado 5 Ago 2015; citado 7 Ago 2015]. Pérdida de peso y apetito (caquexia-anorexia) [aprox. 5 pantallas]. Disponible en:

https://www.aecc.es/SobreElCancer/CuidadosPaliati vos/sintomasmasfrecuentesytratamiento/Paginas/Per didadepesoyapetito.aspx

32. Naranjo Pereira M. Autoestima: un factor relevante en la vida de la persona y tema esencial del proceso educativo. Actualidades Investigativas en Educación [serial de internet: ISNN 1409-4703], 2007 [citado 7 Ago 2015]; 7(3): [aprox. 27 p.] Disponible en: http://revista.inie.ucr.ac.cr/index.php/aie/article/vi ew $/ 683 / 746$

33. Vasquez-Mojerón A, Garía-Bóveda J, VásquezMojerón. Escala de autoestima de Rosenberg: fiabilidad y validez en población clínica española. Apuntes de Psicología [serial de internet: ISSN 0213-3334], 2004 [citado 7 Ago 2015]; 22(2): [aprox. 8 p.] Disponible en:

http://www.apuntesdepsicologia.es/index.php/revista /article/view/53/55

34. Mi Autoestima [Internet]. España: La asociación; c2011 [citado 7 Ago 2015]. Autoestima y trastornos alimentarios [aprox. 2 pantallas]. Disponible en: http://www.miautoestima.com/autoestima-obesidadbulimia-anorexia/

35. Ormaetxea O, La Alexitimia. Tesis doctoral. Barcelona: Instituto Superior de Estudios Psicológicos; 2010

36. Medline Plus [Internet]. EE.UU: La asociación; s.f. [Actualizado 20 Dic 2014; citado 7 Ago 2015]. Autoestima y trastornos alimentarios [aprox. 2 pantallas]. Disponible en:

http://www.miautoestima.com/autoestima-obesidadbulimia-anorexia/

37. Guadarrama-Guadarrama R, Domínguez-Valdez K, López R, Toribio-Pérez L. Anorexia y bulimia y su relación con la depresión en adolescentes. Psico salud. 2008; 18(1): 57-61.

38. Torres V, Chávez A. Ansiedad. Rev. Act. Clin. Med [online]. 2013; (35): 1788-92.

39. Pintado-Cucarella S. Bienestar emocional, imagen corporal, autoestima y sexualidad en mujeres con cáncer de mama. Tesis doctoral. Valencia: Universidad de Valencia, Facultad de Psicología; 2013.

40. Penna-Tosso M, Sánchez M. ABC... anorexia, bulimia y comedor compulsivo-Manual para docentes. $1^{\mathrm{a}}$ edición, Madrid: INUTCAM, s.f. [online] (Citado el 27 Ago 2015).

41. Salud Madrid. [internet]. Madrid: La asociación; s.f. [Citado 08 Ago 2015]. [Aprox. 4 pantallas].

42. ANAD. [Internet]. Naperville: la asociación; c2015 [citado 08 Ago 2015]. [Aprox. 3 pantallas]. Disponible en:

http://www.anad.org/get-information/eating-disordersigns-and-symptoms/

43. SEAIC [Internet]. España: La asociación; c2012 [citado 8 Ago 2015]. Criterios diagnosticos [aprox. 2 pantallas]. Disponible en: http://www.seaic.org/profesionales/criteriosdiagnosticos 
44. Bolaños-R P. Cuestionarios, inventarios y escalas. En: Bolaños-R P, editor Trastornos de la Conducta Alimentaria. Sevilla: ICC; 2013. p. 1981-2007.

45. Urzúa-M A, Castro-R S, Lillo-O A, Leal-P C. Evaluación de los trastornos alimentarios: propiedades psicométricas del test EDI-2 en adolescentes escolarizados(as) de 13 a 18 años. Rev Chil Nutr. 2009; 36 (4): 1063-73

46. Iñarritu-P M, Cruz-L V e Morán-A I. Instrumentos de evaluación para los trastornos de la conducta alimentaria, Rev RESPYN. Julio 2004; 5(2).

47. Morgan J, Reid F, Lacey J. El cuestionario SCOFF: una nueva herramienta de detección de trastornos de la alimentación. West J Med. 2000; 172 (3): 164-65.

48. Sicolog.com [Internet]. España: La asociación; s.f [actualizado 15 ago 2015; citado 29 enero 2016]. Test de Imágen Corporal - Cuestionario B.S.Q. (Body Shape Questionnarire).

49. Castrillón D; Montaño I; Avendaño G; Pérez A. Validación del Body Shape Questionnaire (cuestionario de la figura corporal) BSQ para la población colombiana. Acta Colombiana de Psicología [Revista en Internet]. 2007

50. Esteves-López E, Murgui-Pérez S, Musitu-Ochoa G, Moreno-Ruiz D. Clima familiar, clima escolar y satisfacción con la vida en adolescentes. Rev Mex Psic. 2008; 25(1): 119-28.
51. Jimenez-Talon L, Ferrero M, Gómez R, Parra P. Evaluación del clima familiar en una muestra de adolescentes. Rev de Psicol. Gral y Aplic. 1999; 52(4): 453-62.

52. Mental Health Ministries [Internet]. EE.UU: La asociación; s.f [citado 19 Mar 2016].

53. Herpertz-Dahlmann B, Salbach-Andrae H. Overview of treatment modalities in adolescent anorexia nervosa. Child Adolesc Psychiatr Clin. N Am. 2009; 18 (1): 131-45.

54. Miján de la Torre A, Pérez-García A, Martín de la Torre E, Mateo Silleras B. Is an integral nutritional approach to eating disorders feasible in primary care? Br J Nutr. 2006; 96 (1):82-5.

55. NHS-Quality Improvement Scotland. Eating Disorders in Scotland: Recommendations for management and treatment. Edimburgh: NHS QIS; 2007

56. Jáuregui-LoberaI, Bolaños-Ríos P. Revisión del tratamiento dietético-nutricional de la anorexia nerviosa. Rev Med Chile. 2012; 140: 98-107.

57. National Institute for Health and Clinical Excellence [Internet]. London: La asociación; c2014. Eating disorders: Core interventions in the treatment and management of anorexia nervosa, bulimia nervosa and related eating disorders.

58. Davis H., Attia E. Pharmacotherapy of eating disorders. Curr. Op. Psy. 2017; 30(6):452-57. 
